\title{
Erratum to: Carbon nanostructured materials for applications in nano-medicine, cultural heritage, and electrochemical biosensors
}

\author{
F. Valentini • M. Carbone $\cdot$ G. Palleschi
}

Received: 22 January 2014 / Accepted: 22 January 2014 / Published online: 18 March 2014

(C) Springer-Verlag Berlin Heidelberg 2014

\section{Erratum to: Analytical and Bioanalytical Chemistry DOI 10.1007/s00216-012-6351-6}

Unfortunately, there was a mistake in Fig. 3 of this contribution. The panels $3 \mathrm{a}$ and $\mathrm{b}$ should have been omitted. The correct figure is

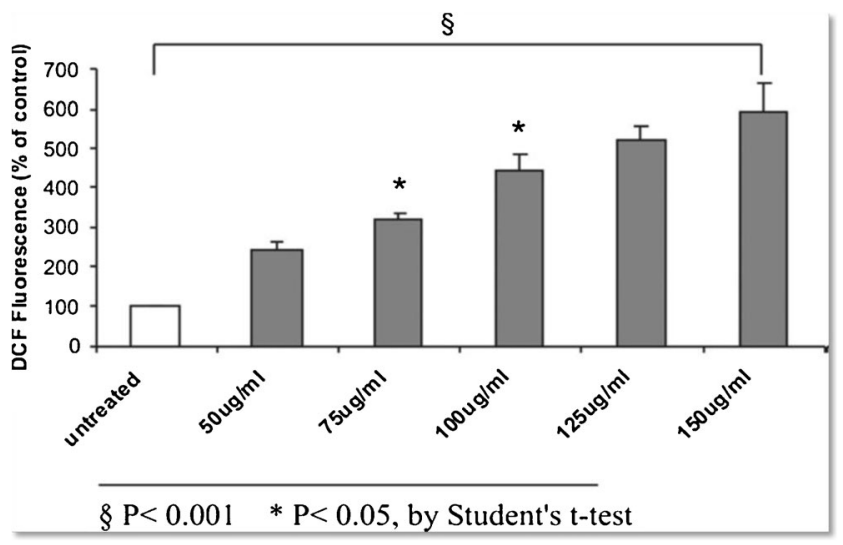

Fig. 3 Induction of ROS generation by SWCNTs in HGFs. Cells were treated with different concentrations of nanotubes $(50,75,100,125$, $150 \mu \mathrm{g} / \mathrm{ml}$ ) for $24 \mathrm{~h}$. The ROS production was measured by analysis of DCF fluorescence compared to untreated cultures. The results are expressed as percentage of control and reported as means \pm SE of four separate experiments for each experimental point. The symbol (§) refers to the comparison between treated and untreated cultures. The symbol $(*)$ refers to the comparison between one single dose and the previous one. (Published from Cicchetti R, Diviza M. Valentini F, Argentin G (2011) Effect of singlewall carbon nanotubes in human cells of the oral cavitiy: Geno-cytotoxic risk. Toxicology in Vitro 25: 1811-1819 with permission of Elsevier Ltd.)

The online version of the original article can be found at http://dx.doi.org/ 10.1007/s00216-012-6351-6.

F. Valentini $(\bowtie) \cdot$ M. Carbone $\cdot$ G. Palleschi

Dipartimento di Scienze e Tecnologie Chimiche, II Università

Degli Studi di Roma Tor Vergata,

via della Ricerca Scientifica 1,

00133 Roma, Italy

e-mail: federica.valentini@uniroma2.it
The authors would also like to thank Prof. Andrea Magrini, Department of Biopathology-Occupational Medicine, Tor Vergata University, and Dr. Luisa Campagnolo of the Department of Biomedicine and Prevention for providing useful material. 